\title{
C. Lösungsansätze zur Organhaftung bei unklarer Rechtslage
}

Die Auslegung hat gezeigt, dass es dogmatisch richtig ist, dem Organträger bei Entscheidungen unter unklarer Rechtslage einen Ermessensspielraum einzuräumen. Abschließend bleibt allerdings ungeklärt, wie der Entscheidungsprozess des Organträgers aussehen muss, um sich rechtssicher im Bereich der Haftungsprivilegierung bewegen zu können. Zwar kann und sollte hier die Vertretbarkeitstheorie als Grundlage dienen. Allerdings wäre es nicht ausreichend $\mathrm{zu}$ sagen, dass die Vertretbarkeitstheorie den gesamten Prozess darstellt. Daher sollen im folgenden Abschnitt, teilweise unter Heranziehung der Vertretbarkeitstheorie, mögliche Lösungsvorschläge, die eine Prozesskontrolle für Entscheidungen unter unklarer Rechtslage umsetzen, dargestellt und abschließend kritisch gewürdigt werden. Dabei wird nicht mehr auf den Prozess bis zur Feststellung einer odd's opinion eingegangen. Es geht hier vielmehr um den Umgang mit der odds‘ opinion selbst.

\section{Lösung: Entwicklung einer Legal Judgement Rule de lege lata?}

Die rechtlich sicherste Möglichkeit, welche gleichzeitig die Ungewissheit auflöst, wäre ein Lösungsansatz basierend auf der aktuellen Rechtslage. Hier käme eine direkte oder analoge Anwendung des $\S 93$ Abs. 1 S. 2 AktG auf Entscheidungen bei unklaren Rechtslagen in Frage.

1. Direkte Anwendung der Business Judgement Rule auf die unklare Rechtslage

In der Literatur anerkannt ist, dass der Tatbestand des $\S 93$ Abs. 1 S. 2 AktG, insbesondere das Tatbestandsmerkmal „unternehmerische Entscheidung“ weit auszulegen ist. Daher werden aufgrund der weiten Begriffsfassung der unternehmerischen Entscheidung de lege lata oftmals auch Entscheidungen unter unklarer Rechtslage unmittelbar unter das Tatbestandsmerkmal subsumiert. ${ }^{264}$ Unternehmerische Entscheidungen sind eben nicht rechtlich gebundene

${ }^{264}$ vgl. nur Buck-Heeb, BB 2013, S. 2251 f.; GK AktG/Hopt/Roth, § 93 Rn. 83.

(C) Der/die Autor(en) 2021

M. Willen, Die Business Judgement Rule, Business, Economics, and Law, https://doi.org/10.1007/978-3-658-31322-7_3 
Entscheidungen, sondern beinhalten Handlungsalternativen. ${ }^{265}$ In Teilen der Literatur wird argumentiert, dass es sich aufgrund der rechtlichen Unsicherheit aus der ex-ante Sicht gerade nicht um eine rechtlich gebundene Entscheidung handele, weil dadurch Handlungsalternativen bestünden, bei denen nicht abschließend geklärt sei, welche Alternative die Rechtsprechung favorisieren würde. Daher sei es falsch, dem Organträger nur deswegen die Haftungsprivilegierung zu versagen, weil es thematisch nicht um eine finanzielle Entscheidung geht, sondern um die Auslegung einer Rechtsnorm. ${ }^{266}$ Bestärkt wird diese Ansicht, dass Teile der Literatur aufgrund der eigenverantwortlichen Unternehmensleitung in der Aktiengesellschaft ( $§ 76 \mathrm{AktG})$ in Verbindung mit dem Grundgedanken der Satzungsstrenge ( $\S 23$ Abs. 5 AktG) annehmen würden, dass alle Regelungsbereiche, die der Gesetzgeber im Aktienrecht explizit nicht ausgestaltet hat, der Unternehmensleitung zu zuordnen seien und somit auch unter die Business Judgement Rule fallen würden. ${ }^{267}$

Die Gegenmeinung vertritt die Auffassung, dass die Anwendung der Business Judgement Rule auf Entscheidungen unter unklarer Rechtslage der Begründung des Gesetzgebers entgegenstünden und daraus eine unzulässige Ausweitung des Tatbestandes resultieren würde. Hier wird der Beweis herangezogen, dass es laut dem Gesetzgeber ,für illegales Verhalten [...] keinen sicheren Hafen“ gäbe ${ }^{268}$ und der Gesetzgeber auch explizit in der Begründung die unternehmerische Entscheidung von rechtlich gebundenen Entscheidung, worunter auch „sonstige

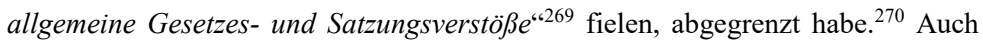
Schäfer behauptet, dass die direkte Anwendung der Business Judgement Rule dem Willen des Gesetzgebers entgegenstünde. Es sei wesentlicher Sinn und Zweck der Business Judgement Rule, vor möglichen Rückschaufehlern zu schützen. Allerdings könne sich hinsichtlich der Auslegung der Rechtslage und den damit verbundenen Alternativen faktisch keine unterschiedliche Beurteilung der

\footnotetext{
${ }^{265}$ vgl. nur Brock, Legalitätsprinzip und Nützlichkeitserwägungen, S. 38; GK AktG/Hopt/Roth, § 93 Rn. 80; Henssler/Strohn/Dauner-Lieb, § 93 AktG Rn. 21; Henssler/Strohn/Oetker, § 43 GmbHG Rn. 27 f.; Hölters/Hölters, § 93 Rn. 30; Hüffer/Koch/Koch, § 93 Rn. 16; MHLS/Ziemons, § 43 Rn. 137 f.; MüKoAktG/Spindler, § 93 Rn. 48.

${ }^{266}$ Buck-Heeb, BB 2013, S. 2251; Kocher, CCZ 2009, S. 217; eine ähnliche Auffassung vertritt auch: Bicker, AG 2014, S. 10; Ebbinghaus/Hasselbach, AG 2014, S. 875.

${ }^{267}$ Bachmann, WM 2015, S. 108 f.; Bachmann, ZIP 2014, S. 580; Brock, Legalitätsprinzip und Nützlichkeitserwägungen, S.196.

${ }^{268}$ Begr. RegE UMAG, BT-Drucks. 15/5092, S. 11.

${ }^{269}$ Begr. RegE UMAG, BT-Drucks. 15/5092, S. 11.

${ }^{270}$ vgl. nur MüKoAktG/Spindler, § 93 Rn. 88; Nietsch, ZGR 2015, S. 642 f.
} 
Rechtslage bei dem Entscheidungsträger und der Rechtsprechung ergeben. Sollte die unternehmerische Entscheidung aufgrund der Rechtsprechung pflichtwidrig sein, so sei die Entscheidung faktisch auch schon zum Entscheidungszeitpunkt pflichtwidrig gewesen. Auf die spätere gerichtliche Beurteilung käme es nicht an. ${ }^{271}$

Der 70. Deutsche Juristentag hat sich ebenfalls mit der Frage beschäftigt, ob eine ausdrückliche Kodifizierung der unklaren Rechtslage in den Tatbestand der Business Judgement Rule erfolgen soll. Eine ausdrückliche Kodifizierung wurde abgelehnt. Auf eine Äußerung hinsichtlich der lex lata wurde jedoch verzichtet. ${ }^{272}$ Schlussendlich spricht dieser Verzicht jedoch argumentativ sowohl für als auch gegen die direkte Anwendung.

Die These gegen eine direkte Anwendung wegen des Widerspruchs zur Gesetzesbegründung könnte dogmatisch gelöst werden, wenn die Business Judgement Rule nicht direkt, sondern analog auf die unklare Rechtlage angewendet werden könnte.

2. Analoge Anwendung der Business Judgement Rule auf die unklare Rechtslage

Um eine Analogie jedoch bejahen zu können, bedarf es einer planwidrigen Regelungslücke hinsichtlich der Entscheidungsfindung bei unklaren Rechtslagen sowie darauf aufbauend eine vergleichbare Interessenslage zwischen den Grundideen der Literatur hinsichtlich der rechtlichen Behandlung von unklaren Rechtslagen und der Business Judgement Rule.

\section{a) Planwidrige Regelungslücke}

Es steht außer Zweifel, dass die Entscheidungsfindung unter unklarer Rechtslage nicht abschließend geklärt ist. Eine Kodifizierung oder Ähnliches sind nicht vorhanden. Ansonsten stünde diese Ausführung der Lösungsfindung auch im

\footnotetext{
${ }^{271}$ Schäfer, ZIP 2005, S. 1256; so auch: Thole, ZHR (173) 2009, S. 523.

272 Deutscher Juristentag, Verhandlungen des 70. Deutschen Juristentages, S. N 62; GK AktG/Hopt/Roth, § 93 Rn. 27.
} 
Widerspruch zur tatsächlichen Rechtslage und würde diese Arbeit größtenteils bedeutungslos machen.

\section{b) Vergleichbare Interessenlage}

$\mathrm{Ob}$ die Interessenslage vergleichbar ist, ist in der Literatur umstritten, wird jedoch von der Mehrheit der Literatur angenommen. ${ }^{273}$ Von vornherein ist festzustellen, dass die Argumente für eine direkte Anwendung der Business Judgement Rule ebenfalls auf die Möglichkeit der analogen Anwendung analog anwendbar sind. ${ }^{274}$ So ist der Zweck der Business Judgement Rule unternehmerisches Handeln, welches aufgrund des Prognosecharakters stets mit Risiken verbunden ist, zu schützen. Die Umsetzung erfolgt, indem nicht der Organträger für Schäden haftet, sondern derjenige, der auch vom Erfolg der Gesellschaft profitiert, also der Gesellschafter oder Aktionär. Die Literatur ist sich insoweit einig, dass dieser Grundsatz auch für Entscheidungen bei unklaren Rechtslagen gelten sollte, da auch hier Risiken wegen des Prognosecharakters drohen würden. ${ }^{275}$ Generell stellt die Literatur fest, dass eine Entscheidung unter unklarer Rechtslage durchaus unter den allgemeinen Tatbestand der unternehmerischen Entscheidung subsumierbar sei. ${ }^{276}$ Zusätzlich führt Buck-Heeb an, dass das Argument, dass es sich bei unklaren Rechtslagen um Fragen der Legalität handele und daher gebundene Entscheidungen seien, schon allein deswegen nicht tragbar sei. Es gäbe keinen sicheren Hafen für offenbar rechtswidriges Verhalten. Dies treffe aber nicht $\mathrm{zu}$, wenn der Entscheidungsträger zwischen Handlungsalternativen unter rechtlicher Unsicherheit eine Alternative auswählen müsse. ${ }^{277}$

Die Gegenauffassung zieht zur Bekräftigung ihres Standpunktes die Gesetzesbegründung zum UMAG heran. Ein weiterer Zweck der Business

\footnotetext{
273 vgl. nur: Bicker, AG 2014, S. 10; Buck-Heeb, BB 2013, S. 2252; Ebbinghaus/Hasselbach, AG 2014, S. 875; GK AktG/Hopt/Roth, § 93 Rn. 140; Graewe/von Harder, BB 2017, S. 711; MüKoAktG/Spindler, § 93 Rn. 89; Spindler/Stilz/Fleischer, § 93 Rn. 32; Thole, ZHR (173) 2009, S. 523.

${ }^{274}$ Hinsichtlich der Argumente für eine direkte Anwendung siehe: Bicker, AG 2014, S. 10; BuckHeeb, BB 2013, S. 2251 f.; Ebbinghaus/Hasselbach, AG 2014, S. 875; GK AktG/Hopt/Roth, § 93 Rn. 83; Kocher, CCZ 2009, S. 217.

${ }^{275}$ vgl. nur Bicker, AG 2014, S. 10; Buck-Heeb, BB 2013, S. 2252; MüKoAktG/Spindler, § 93 Rn. 89; Spindler/Stilz/Fleischer, $\$ 93$ Rn. 32.

${ }^{276}$ Ebbinghaus/Hasselbach, AG 2014, S. 875; GK AktG/Hopt/Roth, 93 Rn. 140; Graewe/von Harder, BB 2017, S. 711; Thole, ZHR (173) 2009, S. 523.

${ }^{277}$ Buck-Heeb, BB 2013, S. 2252 f.
} 
Judgement Rule sei es schließlich, zum Wohle der Gesellschaft die unternehmerische Risikobereitschaft der Organträger $\mathrm{zu}$ fördern. Allerdings entspräche eine Entscheidung unter unklarer Rechtslage nicht diesem Zweck. Vielmehr ginge es hier darum eine generelle Haftungsbefreiung zu realisieren. Auch der Schutzzweck vor Rückschaufehlern stehe im Widerspruch zur Analogie. So ist das Kernelement dieses Schutzzweckes, dass ein Richter ex-post und mit weniger Fachkenntnissen in der unternehmerischen Praxis leicht $\mathrm{zu}$ unterschiedlichen Ansichten gegenüber dem Organträger kommen könne. Bei einer Entscheidung unter unsicherer Rechtslage würde dieses wirtschaftliche durch ein juristisches Merkmal ersetzt, wobei davon auszugehen sei, dass das Gericht zumindest auf dem gleichen Erfahrungs- und Kenntnisniveau mit dem Organträger ist. $^{278}$

Ungeachtet dessen führt die teleologische Auslegung der Business Judgement Rule in der Literatur verbreitet zu dem Ergebnis, dass die (rechtliche) Entscheidung für eine Handlungsalternative objektiv mit der einer unternehmerischen Entscheidung im Sinne der Business Judgement Rule vergleichbar ist, weshalb eine analoge Anwendung realisierbar wäre. ${ }^{279}$ Es bleibt abschließend fraglich, ob die Business Judgement Rule in ihrer Gesamtheit, wegen der Tatsache, dass eine Entscheidung bei unklarer Rechtslage unter den Tatbestand der unternehmerischen Entscheidung subsumiert werden kann, analog anwendbar ist. Unterstützt wird die Skepsis durch die Widersprüche zur Gesetzesbegründung. ${ }^{280}$

Diese Bedenken können hier allerdings ungeklärt bleiben. Denn losgelöst von der Frage der analogen Anwendbarkeit der Business Judgement Rule könnte eine Rechtsfortbildung de lege ferenda unter Beachtung des Meinungsstreits und den neu erworbenen Kenntnissen der Vertretbarkeitstheorie eine dogmatisch korrekte Lösung bringen, da in der Literatur zumindest Einigkeit darüber besteht, dass eine Durchbrechung der Legalitätsbindung bei Entscheidungen unter Unsicherheit notwendig ist. ${ }^{281}$

\footnotetext{
278 Buck-Heeb, BB 2013, S. 2253. So auch: Thole, ZHR (173) 2009, S. 521 f. Gegen eine Anwenbarkeit der Business Judgement Rule als Ganzes sind auch: Brock, Legalitätsprinzip und Nützlichkeitserwägungen, S.199; MüKoAktG/Spindler, § 93 Rn. 88.

${ }^{279}$ Buck-Heeb, BB 2013, S. 2253; Hüffer/Koch/Koch, § 93 Rn. 19; Verse, ZGR 2017, S. 193.

${ }^{280}$ Begr. RegE UMAG, BT-Drucks. 15/5092, S. 11.

${ }^{281}$ Buck-Heeb, BB 2013, S. 2251; Ebbinghaus/Hasselbach, AG 2014, S. 877; Graewe/von Harder, BB 2017, S. 707; Spindler/Stilz/Fleischer, § 93 Rn. 30; Thole, ZHR (173) 2009, S. 521 f.
} 


\section{Lösung: Entwicklung einer Legal Judgement Rule de lege ferenda?}

Inhaltlich gestützt wäre die Rechtsfortbildung überdies auf der Business Judgement Rule. In der dazugehörigen Gesetzesbegründung heißt es, dass „,[d]as für das Aktiengesetz zu \& 93 gefundene Regelungsmuster und die Literatur und Rechtsprechung dazu [...] als Anknüpfungs- und Ausgangspunkt für die weitere Rechtsentwicklung dienen [können]. “282

Von Natur aus Voraussetzung der Rechtsfortbildung ist die Berücksichtigung des Status Quo. Hier sollen zum einen die Business Judgement Rule als Maßstab und Anknüpfungspunkt und zum anderen die aktuelle Rechtsprechung unter Heranziehung der Grundsätze der Vertretbarkeitstheorie dienen.

Dogmatisch losgelöst von der Business Judgement Rule könnte durch die Festsetzung der Vertretbarkeitstheorie als Kontrollinstrument für Entscheidungen unter unklarer Rechtslage der bisherige Meinungsstreit über die Anwendbarkeit der Business Judgement Rule geklärt werden. Denn die Vertretbarkeitstheorie ist weder eine analoge noch direkte Anwendung der Business Judgement Rule. Vielmehr wurde die Business Judgement Rule als Hilfestellung verwendet, um dem gesetzgeberischen Organhaftungsregime zu entsprechen. Dadurch konnte innerhalb der Auslegung dieser Arbeit festgestellt werden, dass die Vertretbarkeitstheorie sowohl dem wortwörtlichen als auch objektiven Willen des Gesetzgebers entspricht, ohne gleichzeitig mit anderem geltenden Recht zu kollidieren. ${ }^{283}$ Die Frage hinsichtlich der Dogmatik und inwiefern die Vertretbarkeitstheorie dem gesetzgeberischen Willen entspricht erübrigt sich daher. Ein weiterer Vorteil der Vertretbarkeitstheorie ist, dass diese dem Organträger den von der Literatur überwiegend unstrittig verlangten Ermessensspielraum zur Entscheidung unter Rechtsunsicherheit zuspricht. Auch die Weite des Ermessensspielraumes ist in beiden Fällen weit zu fassen und durch die mögliche Etablierung der beweglichen Ermessensschranke den Umständen entsprechend im Einzelfall zu betrachten.

\footnotetext{
${ }^{282}$ Begr. RegE UMAG, BT-Drucks. 15/5092, S. 12.

${ }^{283}$ Hinsichtlich der Vertretbarkeitstheorie im Allgemeinen vgl. hierzu S. 45 ff. Hinsichtlich der Auslegung der Legalitätspflicht und der Bestätigung der Vertretbarkeitstheorie vgl. v.A. S. 72.
} 
Im Ergebnis liegt faktisch und bezogen auf die Praxis kein Unterschied zwischen der Business Judgement Rule und der Vertretbarkeitstheorie vor, weshalb der Terminus „Legal Judgement Rule“ zwar nicht wortwörtlich, aber im Kontext durchaus vertretbar wäre.

Zusammenfassend zeigt sich, dass die Vertretbarkeitstheorie das Dilemma der direkten oder analogen Anwendung der Business Judgement Rule auf Entscheidungen unter unklaren Rechtslagen löst, indem die Business Judgement Rule nur als Anknüpfungspunkt dient. Aus der Gesetzesbegründung zu $\S 93$ Abs. 1 S. 2 AktG ergibt sich, dass die Anknüpfung und darauf aufbauende Rechtsfortbildungen nicht nur gewährt, sondern gewollt wird. Ein Widerspruch zum objektiven Willen des Gesetzgebers ist also nicht ersichtlich. Gleichzeitig erfüllt die Vertretbarkeitstheorie auch die von der überwiegenden Literatur verlangte Durchbrechung der Legalitätspflicht und Haftungsprivilegierung der Entscheidungsträger durch die Anwendung eines Ermessensspielraumes bei Entscheidungen unter Rechtsunsicherheit. Abschließend ist hier ebenfalls festzustellen, dass so die Durchführung einer Prozesskontrolle bei unklaren Rechtslagen und gleichzeitig eine Verortung des Beurteilungsspielraumes vom Verschulden hin zur Pflichtwidrigkeit möglich wäre.

\section{Lösung: Status Quo als ausreichender Schutz}

Im Umkehrschluss stellt dies dann allerdings auch einen Widerspruch zur aktuellen Rechtsprechung und Teilen der Literatur dar, die den Beurteilungsspielraum innerhalb des Verschuldens sehen. Danach sei die Entscheidung unter unklarer Rechtslage lediglich ein Haftungsproblem, welches es besser auf der Verschuldensebene zu klären gilt. ${ }^{284}$ Eine ähnliche Auffassung liefert auch die Regierungsbegründung. Danach kann ,im Sinne einer haftungstatbestandlichen Freistellung [...] es [...] hier im Einzelfall aber am Verschulden fehlen. "285 Nach dem Ansatz der Lösung auf der Verschuldensebene wäre bei einer Entscheidung

${ }^{284}$ Binder, AG 2012, S. 890, 895; Buck-Heeb, BB 2013, S. 2254; Hüffer/Koch/Koch, § 93 Rn. 19; MüKoAktG/Spindler, § 93 Rn. 89; Spindler/Stilz/Fleischer, § 93 Rn. 32. Die Argumentation der Literatur basiert dabei stets auf dem von der Rechtsprechung in Sachen ISION entwickelten Schema: $B G H$, NZG 2011, S. 1272 f.; $B G H$, NZG 2015, S. 794 f.

${ }^{285}$ Begr. RegE UMAG, BT-Drucks. 15/5092, S. 11. 
unter Rechtsunsicherheit, die sich nachträglich durch die Rechtsprechung als falsch herausstellt, stets die vorsätzliche Pflichtwidrigkeit $\mathrm{zu}$ bestätigen, denn der Entscheidungsträger hat wissentlich und gewollt die Entscheidung getroffen und durchgeführt. Erst auf der Ebene des Verschuldens wäre dann eine Haftung des Entscheidungsträgers bei unklarer Rechtslage abzulehnen, wenn der Entscheidungsträger sich im Verbotsirrtum über die unklare Rechtslage befunden hätte. ${ }^{286}$ Für die Erfüllung des Tatbestandes des Verbotsirrtums wird vom BGH vorausgesetzt, dass ,,[...] der Täter alle seine geistigen Erkenntniskräfte [einsetzt] und etwa aufkommende Zweifel [...] durch Einholung [eines] verlässlichen und sachkundigen Rechtsrats beseitigt [...]. [...] [Die] Auskunft selbst muss zudem einen unrechtsverneinenden Inhalt haben. " 287

Insofern besteht zwar auch hier ein Beurteilungsspielraum des Entscheidungsträgers, welcher durch einen fundierten Rechtsrat bestätigt werden muss. Die Konsequenz einer gerichtlichen Gegenentscheidung bei unklarer Rechtslage ist jedoch zu allererst die Pflichtwidrigkeit des Organträgers, welche wiederum andere Rechtsfolgen hervorbringen könnte. Zwar gibt es Stimmen in der Literatur, die andere mögliche Rechtsfolgen, wie die Abberufung des Organträgers oder die Möglichkeit der Anfechtung des Entlastungsbeschlusses, verneinen, ${ }^{288}$ allerdings gibt es dazu auch gegenteilige Auffassungen ${ }^{289}$. Eine abschließende Aussage kann aus diesem Grund nicht getroffen werden, weshalb das Risiko für den Organträger hier vorsorglich stehen bleiben muss. Dessen ungeachtet bleibt die Möglichkeit bestehen, dieses Risiko durch den Abschluss einer D\&O-Versicherung faktisch einzuschränken bzw. bedeutungslos zu gestalten, indem der tatsächliche Schaden, bis auf den Selbstbehalt, von der Versicherungsgesellschaft getragen wird. ${ }^{290}$ Allerdings bleibt dabei die Pflichtwidrigkeit dogmatisch erhalten, weshalb dieser

\footnotetext{
${ }^{286}$ Buck-Heeb, BB 2013, S. 2252; Ebbinghaus/Hasselbach, AG 2014, S. 874; Lackner/Kühl/Kühl, $\S 17$ Rn. 7; Schönke/Schröder/Sternberg-Lieben/Schuster, § 17 Rn. 1; Spindler/Stilz/Hefendehl, § 399 Rn. 271.

${ }^{287}$ BGH, NStZ 2017, S. 284. Darauf aufbauend: MAH AktienR /Ritter/Schüppen, § 24 Rn. 164.

${ }^{288}$ vgl. nur Binder, AG 2012, S. 887 f.; Buck-Heeb, BB 2013, S. 2254; Paefgen, AG 2014, S. 559 f.; Verse, ZGR 2017, S. 192.

${ }^{289}$ vgl. nur GK AktG/Hopt/Roth, § 93 Rn. 140; Holle, AG 2016, S. 271; Spindler/Stilz/Fleischer, § 93 Rn. 32.

${ }^{290}$ Zur D\&O-Versicherung im Allgemeinen vgl. S. 35 f. Ansonsten: Cyrus, NZG 2018, S. 9; Grigoleit/Grigoleit/Tomasic, § 93 Rn. 95; Hölters/Hölters, § 93 Rn. 398; 401; Hüffer/Koch/Koch, § 93 Rn. 58.
} 
Ansatz, aufgrund der Bedeutung für diese Arbeit, an dieser Stelle außer Acht zu lassen ist.

Zudem wird die Lösung des Konflikts auf der Verschuldensebene dogmatisch nicht sauber gelöst. Der Entscheidungs- und Organträger würde danach in Fällen der Rechtsunsicherheit vollkommen losgelöst von seinem Verhalten und Vorgehen immer pflichtwidrig handeln, wenn er eine Handlungsalternative wählt, die nicht der später folgenden Rechtsprechung entspricht. Denn die gewählte Rechtsansicht bzw. Handlungsalternative muss einen unrechtsverneinenden Inhalt haben. Allerdings ist dies gerade bei Entscheidungen unter Rechtsunsicherheit oft nicht möglich. Zugleich wird die Wahrscheinlichkeit der späteren Auswahl einer Rechtsansicht durch die Rechtsprechung vollkommen außer Acht gelassen. Das bedeutet, dass der Entscheidungsträger zuvor sich noch so sicher sein kann, dass die Rechtsprechung eine Meinung nicht annimmt. Falls sich das im Nachhinein als falsch herausstellen sollte, kann man objektiv gegen den Entscheidungsträger argumentieren, dass er sich eben doch nicht im Verbotsirrtum befand. Einen Schutzmechanismus für den Entscheidungsträger, wie die ,hindsight bias“, gibt es im Strafrecht nicht. Da die eigentliche Handlung des Entscheidungsträgers zu seiner Auswahl folglich unbeachtet bleibt, werden Parallelen zu einer Erfolgshaftung erkennbar. Jedoch ist der objektive Wille des Gesetzgebers gerade nicht der Fokus auf den Erfolg, sondern auf der Handlung, die zum Erfolg führte.

Es gilt zu resümieren, dass objektiv betrachtet der Status Quo zwar eine Haftungsprivilegierung des Organträgers bei Entscheidungen unter unklarer Rechtslage ermöglicht. Jedoch zeigt der Status Quo wesentliche Defizite auf. Durch die Verortung des Beurteilungsspielraumes in das Verschulden und der damit verbundenen Tatbestandsverwirklichung der Rechtswidrigkeit bleibt ein Risiko bestehen, dass der Organträger mit weiteren rechtlichen Konsequenzen zu rechnen hat. Beispielhaft ist hier die Abberufung oder die Anfechtung des Entlastungsbeschlusses zu nennen. Gleichzeitig birgt die Tatsache, dass keine klare Grenze der Haftungsprivilegierung bei Entscheidungen unter Rechtsunsicherheit erkennbar ist, Risiken für den Entscheidungsträger. Beide Risiken resultieren vor allem aus der unsauberen Dogmatik durch die Verortung des Beurteilungsspielraumes. 


\section{Zwischenfazit: Stellungnahme zu den Lösungsansätzen}

Die derzeitige Rechtsunsicherheit hinsichtlich Entscheidungen unter Rechtsunsicherheit und die damit verbundene Haftungsproblematik ist insofern von allen Lösungsansätzen festgestellt worden, als das jeder Ansatz eine Lösung durch Etablierung eines Beurteilungsspielraumes zu finden versucht.

Sowohl die direkte als auch die analoge Anwendung der Business Judgement Rule sind in der Literatur berechtigterweise umstritten. Es gibt sowohl vertretbare Argumente für als auch gegen eine direkte bzw. analoge Anwendung der Business Judgement Rule auf Entscheidungen unter unsicherer Rechtslage. Im Vergleich zwischen der direkten und der analogen Anwendung ist dogmatisch die analoge Anwendung vorzugswürdig, da so ein Widerspruch mit der Gesetzesbegründung vermieden werden kann. Dessen ungeachtet sind beide Theorien in der Literatur umstritten. Weder der Deutsche Juristentag noch die Rechtsprechung oder der Gesetzgeber selbst haben sich bisher konkret zu dem in der Literatur herrschenden Meinungsstreit geäußert. Um eine der beiden Lösungsansätze jedoch vertretbar durchsetzen zu können, müsste über deren Anwendbarkeit Einigkeit bestehen, die so nur durch die Rechtsprechung oder den Gesetzgeber selbst erzielt werden kann. Solange dies nicht geschehen ist, sind beide Theorien abzulehnen. Ansonsten bleibt die Gefahr von erheblichen Rechtsrisiken für die Organträger bestehen.

Nichtsdestotrotz ist beiden Theorien eine wesentliche Erkenntnis zu entnehmen. Sowohl die analoge als auch die direkte Anwendung verlangen eine Durchbrechung der Legalitätsbindung durch Festsetzung eines Beurteilungsspielraumes für den Entscheidungsträger. Diese Vorgehensweise entspricht dem Ansatz der Business Judgement Rule und ist daher dogmatisch sinnvoll und in der Literatur auch unbestritten.

Der gegenwärtige Lösungsansatz durchbricht zwar die Legalitätsbindung des Entscheidungsträgers, indem ein Beurteilungsspielraum gewährt wird, allerdings wird dieser Beurteilungsspielraum in die Ebene des Verschuldens verortet, wodurch Rechtsrisiken beim Entscheidungsträger bestehen bleiben. Da der hier gesuchte Lösungsansatz aber gerade eine Haftungsprivilegierung des Entscheidungsträgers bei Einhaltung eines bestimmten Prozesses regeln soll, ist eine dementsprechende Anwendung fraglich. Zudem bleibt für den Entscheidungsträger auch ungeklärt, wie sein Verhalten praktisch prozessual 
aussehen müsste, um sich im Bereich des Beurteilungsspielraumes zu bewegen. Insofern ist diese Theorie auch nicht hilfreich hinsichtlich des Ziels dieser Arbeit.

Somit bleibt nur der Lösungsansatz durch Rechtsfortbildung übrig, indem die Vertretbarkeitstheorie, de facto als Legal Judgement Rule, zur Lösung herangezogen wird. Da die Vertretbarkeitstheorie dem gesetzgeberischen Willen entspricht, indem sie objektiv in weiten Teilen mit der Business Judgement Rule übereinstimmt und auch in keinem unmittelbaren Bezug zu geltendem Recht steht, gibt es dogmatisch zunächst keine Einwände. Die Auslegung in dieser Arbeit hat gezeigt, dass keine rechtsdogmatischen Widersprüche dieser Theorie erkennbar sind. Zudem durchbricht die Vertretbarkeitstheorie ebenfalls die Strenge der Legalitätspflicht, indem dem Entscheidungsträger ein Beurteilungsspielraum, ähnlich wie der der Business Judgement Rule, zugesprochen wird. Wesentlicher Vorteil gegenüber der gegenwärtigen Rechtslage ist, dass der Beurteilungsspielraum schon innerhalb der Pflichtwidrigkeit selbst ansässig ist. Weitere Risiken des Entscheidungsträgers sind dadurch grundsätzlich ausgeschlossen. Gleichzeitig entspricht es dogmatisch eher der gesetzgeberisch gewollten handlungsorientierten Haftungssystematik.

Gegen diesen Lösungsansatz wiederum spricht, dass der derzeitige Literatur- und Rechtsprechungsstand im Bereich der Vertretbarkeitstheorie übersichtlich und vage ist. Zwar gibt es vereinzelt Bezugnahmen und Ausführungen zu der Vertretbarkeitstheorie $^{291}$, eine tatsächliche Festlegung als Lösungsansatz bleibt derzeit aber aus. Abschließend ungeklärt bleibt daher auch die tatsächliche Rechtsgrundlage. Fraglich ist daher, ob und inwiefern der Vertretbarkeitstheorie tatsächlich zukünftig eine praktische Bedeutung als Lösungsansatz für Entscheidungen unter Rechtsunsicherheit zugesprochen wird. Allerdings fokussiert sich diese Arbeit auf die theoretische Anwendbarkeit der Vertretbarkeitstheorie. Eine Ablehnung aufgrund des geringen Kenntnis- und Forschungsstand in der Wissenschaft wäre daher nicht zielführend.

\footnotetext{
${ }^{291}$ Hier ist vor allem die in dieser Arbeit herangezogene Literatur zur Vertretbarkeitstheorie zu nennen: Brock, Legalitätsprinzip und Nützlichkeitserwägungen, S. 204; Buck-Heeb, BB 2013, S. 2250; Graewe/von Harder, BB 2017, S. 710 f.; Langenbucher, ZBB 2013, S. 22; Spindler/Stilz/Fleischer, § 93 Rn. 30; Thole, ZHR (173) 2009, S. 522 f.
} 
Gemäß dieser Arbeit bleibt rein objektiv die Vertretbarkeitstheorie die sinnvollste und dogmatisch schlüssigste Theorie zur Behandlung von Entscheidungen unter Rechtsunsicherheit.

Open Access Dieses Kapitel wird unter der Creative Commons Namensnennung 4.0 International Lizenz (http://creativecommons.org/licenses/by/4.0/ deed.de) veröffentlicht, welche die Nutzung, Vervielfältigung, Bearbeitung, Verbreitung und Wiedergabe in jeglichem Medium und Format erlaubt, sofern Sie den/die ursprünglichen Autor(en) und die Quelle ordnungsgemäß nennen, einen Link zur Creative Commons Lizenz beifügen und angeben, ob Änderungen vorgenommen wurden.

Die in diesem Kapitel enthaltenen Bilder und sonstiges Drittmaterial unterliegen ebenfalls der genannten Creative Commons Lizenz, sofern sich aus der Abbildungslegende nichts anderes ergibt. Sofern das betreffende Material nicht unter der genannten Creative Commons Lizenz steht und die betreffende Handlung nicht nach gesetzlichen Vorschriften erlaubt ist, ist für die oben aufgeführten Weiterverwendungen des Materials die Einwilligung des jeweiligen Rechteinhabers einzuholen.

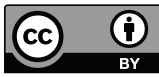

